\title{
The use of taxon-specific reference databases compromises metagenomic classification
}

\author{
Vanessa R. Marcelino ${ }^{1,2,3^{*}}$ D, Edward C. Holmes ${ }^{1,3}$ and Tania C. Sorrell ${ }^{1,2}$
}

\begin{abstract}
A recent article in BMC Genomics describes a new bioinformatics tool, HumanMycobiomeScan, to classify fungal taxa in metagenomic samples. This tool was used to characterize the gut mycobiome of hunter-gatherers and Western populations, resulting in the identification of a range of fungal species in the vast majority of samples. In the HumanMycobiomeScan pipeline, sequence reads are mapped against a reference database containing fungal genome sequences only. We argue that using reference databases comprised of a single taxonomic group leads to an unacceptably high number of false-positives due to: (i) mapping to conserved genetic regions in reference genomes, and (ii) sequence contamination in the assembled reference genomes. To demonstrate this, we replaced the HumanMycobiomeScan's fungal reference database with one containing genome sequences of amphibians and reptiles and re-analysed their case study. The classification pipeline recovered all species present in the reference database, revealing turtles (Geoemydidae), bull frogs (Pyxicephalidae) and snakes (Colubridae) as the most abundant herpetological taxa in the human gut. We also re-analysed their case study using a kingdom-agnostic pipeline. This revealed that while the gut of hunter-gatherers and Western subjects may be colonized by a range of microbial eukaryotes, only three fungal families were retrieved. These results highlight the pitfalls of using taxonspecific reference databases for metagenome classification, even when they are comprised of curated whole genome data. We propose that databases containing all domains of life provide the most suitable option for metagenomic species profiling, especially when targeting microbial eukaryotes.
\end{abstract}

Keywords: Reference database, Fungi, Microbiome, Metagenomic classifier, Misclassification, Assembly errors

\section{Background}

Identifying organisms in metagenome samples remains challenging, especially when working with microbial eukaryotes. Current methods for taxonomic identification of metagenomes can be classified into two categories: assembly-based approaches (yielding metagenome-assembled genomes) and read- (or k-mer) mapping approaches. Assembly-based methods have enabled the discovery and accurate taxonomic identification of a range of bacteria and archaea (e.g. [1]), viruses (e.g. [2]) and, more recently, eukaryotes [3]. Obtaining eukaryotic metagenome-assembled genomes

\footnotetext{
* Correspondence: vrmarcelino@gmail.com

${ }^{1}$ Marie Bashir Institute for Infectious Diseases and Biosecurity and Faculty of Medicine and Health, Sydney Medical School, Westmead Clinical School, The University of Sydney, Sydney, NSW 2006, Australia

${ }^{2}$ Centre for Infectious Diseases and Microbiology, Westmead Institute for Medical Research, Westmead, NSW 2145, Australia

Full list of author information is available at the end of the article
}

requires very deep sequencing, given their large genome sizes and typically low relative abundances. Mapping short sequence reads directly to a reference database is a more sensitive approach to detect rare microorganisms and is usually preferred for taxonomic profiling and comparative studies (reviewed in [4]).

A recent article by Soverini and colleagues [5] describes HumanMycobiomeScan - a new bioinformatics tool to identify fungi in metagenome samples based on mapping sequence reads to a reference database of fungal genomes. The authors applied the pipeline to characterize the gut mycobiome of human populations on different diets (hunter-gatherers and Western populations), resulting in the identification of a range of fungal taxa in 37 of 38 samples analysed. Briefly, the pipeline consists of aligning short sequence reads to a reference database with Bowtie2 [6], filtering human and

(c) The Author(s). 2020 Open Access This article is distributed under the terms of the Creative Commons Attribution 4.0 International License (http://creativecommons.org/licenses/by/4.0/), which permits unrestricted use, distribution, and 
bacterial reads with BMTagger [7], and re-mapping the high-quality reads to a fungal reference database to obtain taxonomic assignments and abundance estimates. The default reference database contains the 66 complete fungal genomes available in NCBI in 2018. Similar taxon-specific reference databases have been implemented in other metagenome classification tools. The FindFungi pipeline for example [8] is based on a Kraken [9] reference database composed of 949 fungal genomes.

The choice of reference database directly impacts diversity and composition inferences from metagenome data [10]. We argue that using taxon-specific reference databases in assembly-free metagenome classification pipelines, such as implemented in HumanMycobiomeScan, leads to an unacceptably high number of misclassifications. Here we demonstrate and discuss the pitfalls of using databases constrained to specific taxon groups, and propose that kingdom-agnostic approaches provide a more accurate metagenome classification.

\section{Analyses and results}

We re-analysed the 38 metagenomes used as a case study in the HumanMycobiomeScan publication [5]. These were obtained from stool samples of Hadza hunter-gatherers from Tanzania $(n=27)$ and Western subjects from Italy $(n=11)$ [11].

To demonstrate the potential pitfalls of using a taxonspecific reference database, we replaced the fungal database in the HumanMycobiomeScan pipeline with a database containing organisms that do not normally occur in the human gut (Additional file 1). Specifically, we built a reference database containing genomes of 18 species of amphibians (frogs and salamanders) and reptiles (snakes, lizards, geckos, turtles and crocodiles). Default values were used for all other HumanMycobiomeScan parameters (see Additional file 2 for details).

HumanMycobiomeScan identified a range of amphibians and reptiles in all the samples analysed, with turtles (Geoemydidae), bull frogs (Pyxicephalidae) and snakes (Colubridae) suggested to be the most abundant herpetological taxa in the human gut (Fig. 1). Remarkably, all species in the reference database were identified in 12 or more samples. The frog species Pyxicephalus adspersus and the snake species Pantherophis guttatus were identified in all samples tested. These results are comparable to the findings of Soverini and colleagues, where 65 fungal species were retrieved when using a database containing 66 fungal genomes [5].

We also analysed the case study using the entire NCBI nucleotide collection (NCBI nt) as a reference and the kingdom-agnostic CCMetagen metagenomic pipeline [12]. Instead of making taxonomic inferences based on individual sequence reads as is done with Bowtie, the CCMetagen pipeline uses KMA [13], which takes advantage of the information from all high-scoring read mappings to determine which reference sequence is the most likely match. The analysis revealed a range of bacteria, archaea, viruses (bacteriophage) and eukaryotes (Additional file 3). Among the eukaryotes identified, we observed obvious misclassifications, including insects, mollusks and plants, which may reflect misidentifications in the NCBI nt reference database [14]. To focus

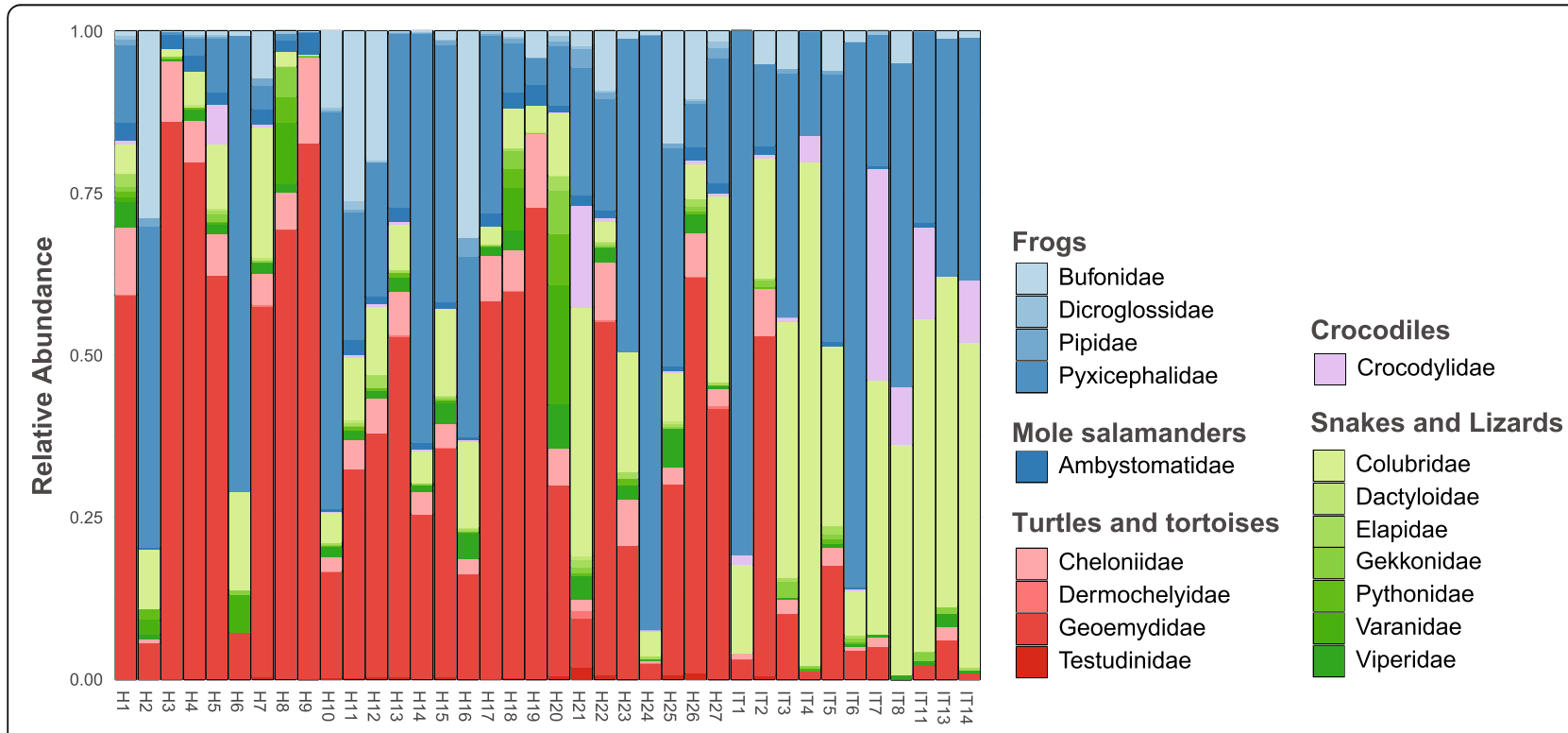

Fig. 1 Herpetofaunal diversity identified in human stool samples of Hadza hunter-gatherers and Western populations using the HumanMycobiomeScan pipeline with a reference database containing genomes of amphibians and reptiles, demonstrating the pitfalls of using taxon-specific reference databases 
on microbial eukaryotes, we filtered out taxonomic assignments belonging to the Arthropoda, Mollusca, Streptophyta, Chordata (including humans), Bacteria, Archaea and viruses (Additional file 2). From these data we detected microbial eukaryotes in 21 subjects. Fungi were detected in only three hunter-gatherers (Fig. 2), comprising Saccharomycetes, Mucoromycetes (Rhizopodaceae) and Dothideomycetes. Species-level identifications could not be retrieved for any of these fungal taxa. Saccharomycetes was the most abundant fungal class with 36 sequence reads. The remaining two fungal taxa were present at extremely low abundance, with only two sequence reads assigned to Mucoromycetes and a single read assigned to Dothideomycetes, making it possible that they represent misclassifications. We additionally retrieved a range of microbial eukaryotes known to colonize the human gut, including the protozoan Blastocystis, parasitic worms (Platyhelminthes and Nematodes), Amoebae and Apicomplexans (Fig. 2). We note, however, that it is likely that some of these also represent false-positives due to contamination in reference databases.

We further investigated the causes of misclassifications by attempting to identify the origins of sequences classified as fungi by HumanMycobiomeScan. Specifically, we analyzed the 101 sequences from sample $\mathrm{H} 1$ belonging to fungi (25 fungal genera) according to HumanMycobiomeScan. Using BLAST [15], we found that $85 \%$ of these putative fungal sequences were rRNA genes (Additional files 2 and 4) that are highly conserved across domains of life. Part of the sequences aligned to unspecified genomic regions (11\%), and only $4 \%$ to protein-coding genes. Of the $101 \mathrm{pu}$ tative fungal sequences, only one was also classified by CCMetagen: this sequence was identified as Blastocysts sp. rRNA by both CCMetagen and BLAST analyses.

\section{Discussion}

Here, we show that using taxon-specific reference databases in assembly-free taxonomic profiling, such as in the HumanMycobiomeScan, can lead to identification of organisms that are demonstrably not present in a metagenome. Although the HumanMycobiomeScan pipeline includes a quality-control step to remove bacterial and human sequences, this is performed after the initial taxonomic assignment and does not prevent misclassifications. The most likely causes of this high number of falsepositives are conserved genetic regions in reference genomes as well as 'contamination' of genome assemblies.

Genes involved in fundamental biological processes change little over evolutionary time and across taxa, and homologues of some of the highly conserved genes, such as those that encode rRNA, are found in all eukaryotes. In assembly-free approaches sequence reads are aligned to the closest (usually homologous) reference sequence in the database, which can be a conserved gene from a distantlyrelated taxon. It is possible that some of the fungi identified by Soverini and colleagues are misclassifications of other abundant microbial eukaryotes in those samples, such as the protozoan Blastocystis. This misclassification problem is

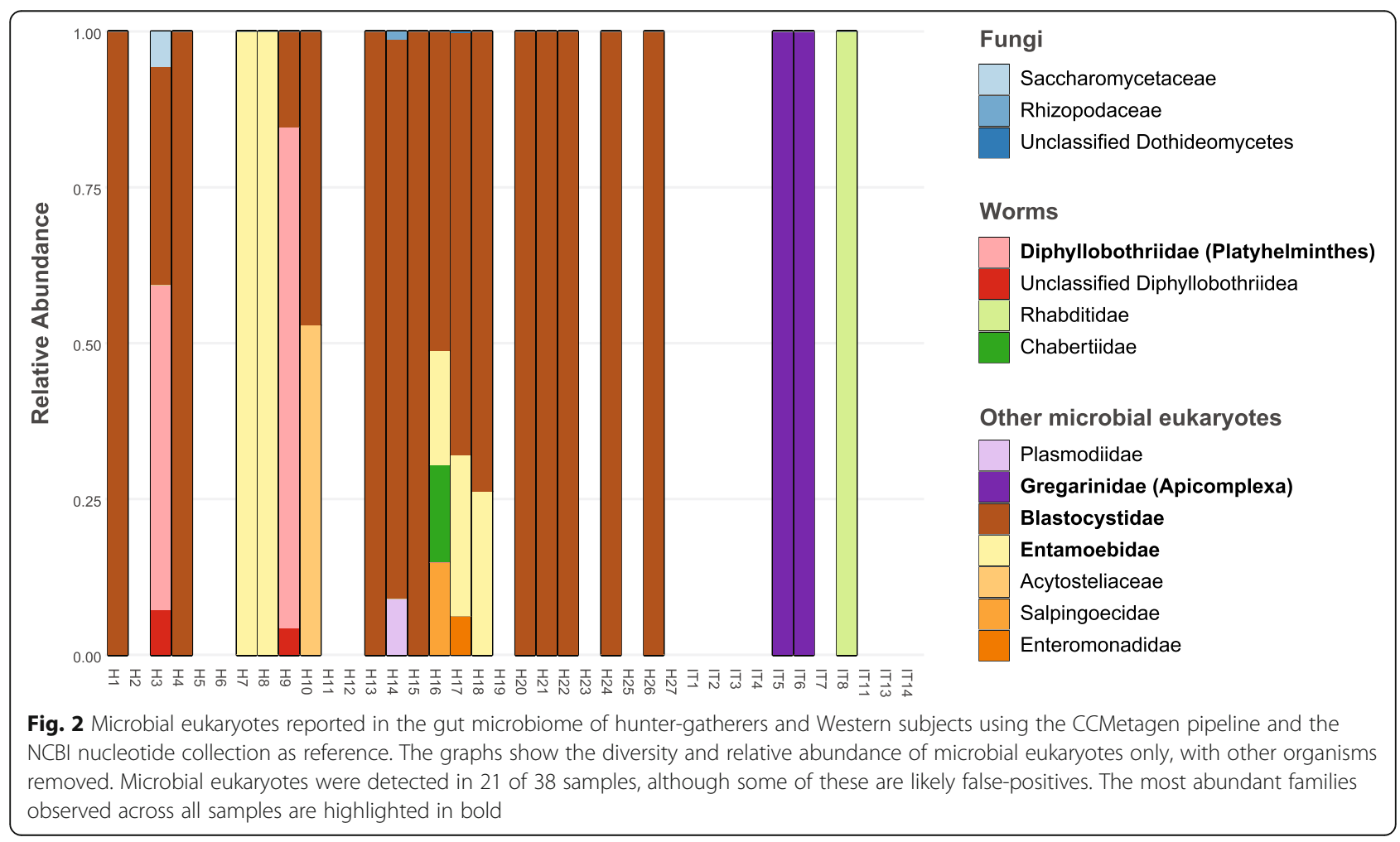


reduced when using reference databases containing a broad range of organisms, such as the NCBI nucleotide collection. Another option to mitigate this issue is to use tools that group all sequence reads that map to the same reference sequence, forming 'pseudo-assemblies' that may span beyond conserved genetic regions $[12,13,16]$.

Reference genome sequences can also be 'contaminated' with bacterial, viral and human DNA, which are commonly sequenced alongside the organism of interest in highthroughput sequencing assays. These contaminant sequences can then be inadvertently assembled with the genome of the target organism. For example, over $10 \%$ of NCBI's genome assemblies of non-primate species are contaminated with human DNA [17]. Some of these contaminants contain open reading frames, with a recent study showing that over 3000 spurious proteins originated from human contaminant sequences have been assigned to bacterial, archaeal and non-human eukaryotic organisms and deposited as such in public databases [18]. Eukaryotic genome assemblies, even those from well-studied organisms, are also commonly contaminated with bacterial DNA: over a hundred bacterial contigs were identified in the genomes of the domestic cow Bos taurus and the model alga Chlamydomonas reinhardtii [19]. Contaminating sequences in assembled genomes often derive from highly abundant genetic elements (e.g. short interspersed nuclear elements and repeats in the human genome) and common contaminants of laboratory reagents, both of which are also likely to be present in metagenome data $[17,18,20]$. We therefore expect that part of the false-positives derive from human and bacterial sequence reads that align to contaminants in reference databases. Contamination and misidentifications compromise a range of reference databases (including NCBI's nt database), and in so doing compromise the results of all metagenome classification tools, including BLAST and CCMetagen. This problem is exacerbated when using reference databases composed of assembled genomes of a single taxonomic group, as a portion of the metagenome sequence reads have no option but to align to the contaminant reference sequence

This serves as a cautionary tale highlighting the pitfalls of using taxon-specific reference databases in assembly-free metagenome classification pipelines. We show that using a reference database restricted to fungal genomes leads to unrealistic taxonomic classifications, and that reptiles and amphibians can also be incorrectly identified in the human gut if a similarly restricted herpetological database is used. Using the NCBI nucleotide collection as reference and our kingdom-agnostic pipeline, we detected a range of microbial eukaryotes - although only three putative fungal taxa - in the metagenomes of Hadza hunter-gatherers and Western individuals. We therefore recommend using databases containing all domains of life for metagenomic species profiling, even if the focus is on particular taxonomic groups.

\section{Supplementary information}

Supplementary information accompanies this paper at https://doi.org/10. 1186/s12864-020-6592-2.

Additional file 1. Genomes of amphibians and reptiles used to construct the herpetofaunal reference database.

Additional file 2. Supplementary Materials and Methods.

Additional file 3. Taxonomic profiling of metagenome samples from hunter-gatherers and Western populations using the CCMetagen pipeline.

Additional file 4. BLAST results of putative fungal sequences identified with HumanMycobiomeScan in sample $\mathrm{H} 1$.

\section{Abbreviations}

NCBI: National center for biotechnology information

\section{Acknowledgments}

We thank the High Performance Computing support and the Research Data Support at the University of Sydney.

Authors' contributions

VRM, ECH and TCS conceptualised the correspondence. VRM performed the analyses. All authors contributed to the manuscript writing. All authors read and approved the final manuscript.

\section{Funding}

This study was funded by an NHMRC Centre of Excellence grant \# APP1102962. ECH is funded by an Australian Laurate Fellowship

(FL170100022). VRM and TCS are Sydney Medical School Foundation Fellows.

\section{Availability of data and materials}

CCMetagen source code is freely available from https://github.com/ vrmarcelino/CCMetagen.

The indexed reference database used with KMA and CCMetagen

(ncbi_nt_no_env_11jun2019) can be downloaded from https://doi.org/10.

$25910 / 5 c c 7 c d 40 f c a 8 e$ and from its mirror at http://www.cbs.dtu.dk/public/

CGE/databases/CCMetagen/.

Ethics approval and consent to participate

Not applicable.

Consent for publication

Not applicable.

\section{Competing interests}

The authors declare that they have no competing interests.

\section{Author details}

${ }^{1}$ Marie Bashir Institute for Infectious Diseases and Biosecurity and Faculty of Medicine and Health, Sydney Medical School, Westmead Clinical School, The University of Sydney, Sydney, NSW 2006, Australia. ${ }^{2}$ Centre for Infectious Diseases and Microbiology, Westmead Institute for Medical Research, Westmead, NSW 2145, Australia. ${ }^{3}$ School of Life \& Environmental Sciences, Charles Perkins Centre, The University of Sydney, Sydney, NSW 2006, Australia.

Received: 17 September 2019 Accepted: 19 February 2020

Published online: 27 February 2020

\section{References}

1. Parks DH, Rinke C, Chuvochina M, Chaumeil PA, Woodcroft BJ, Evans PN, Hugenholtz P, Tyson GW. Recovery of nearly 8,000 metagenome-assembled genomes substantially expands the tree of life. Nat Microbiol. 2017;2:153342.

2. Shi M, Lin XD, Tian JH, Chen LJ, Chen X, Li CX, Qin XC, Li J, Cao JP, Eden JS, et al. Redefining the invertebrate RNA virosphere. Nature. 2016;540:539-43.

3. Olm MR, West PT, Brooks B, Firek BA, Baker R, Morowitz MJ, Banfield JF. Genome-resolved metagenomics of eukaryotic populations during early 
colonization of premature infants and in hospital rooms. Microbiome. 2019; 7:26.

4. Breitwieser FP, Lu J, Salzberg SL. A review of methods and databases for metagenomic classification and assembly. Brief Bioinform. 2017;20:1125-36.

5. Soverini M, Turroni S, Biagi E, Brigidi P, Candela M, Rampelli S. HumanMycobiomeScan: a new bioinformatics tool for the characterization of the fungal fraction in metagenomic samples. BMC Genomics. 2019;20: 496.

6. Langmead B, Salzberg SL. Fast gapped-read alignment with bowtie 2. Nat Methods. 2012;9:357-9.

7. BMTagger: Best Match Tagger for removing human reads from metagenomics datasets. ftp://ftp.ncbi.nlm.nih.gov/pub/agarwala/bmtagger/. Accessed 15 July 2019.

8. Donovan PD, Gonzalez G, Higgins DG, Butler G, Ito K. Identification of fungi in shotgun metagenomics datasets. PLoS One. 2018;13:e0192898.

9. Wood DE, Salzberg SL. Kraken: ultrafast metagenomic sequence classification using exact alignments. Genome Biol. 2016;15:R46.

10. Méric G, Wick RR, Watts SC, Holt KE, Inouye M. Correcting index databases improves metagenomic studies. bioRxiv. 2019. https://doi.org/10.1101/ 712166

11. Rampelli S, Schnorr SL, Consolandi C, Turroni S, Severgnini M, Peano C, Brigidi P, Crittenden AN, Henry AG, Candela M. Metagenome sequencing of the Hadza hunter-gatherer gut microbiota. Curr Biol. 2015;25:1682-93.

12. Marcelino VR, Clausen PT, Buchman J, Wille M, Iredell JR, Meyer W, Lund O, Sorrell T, Holmes EC. CCMetagen: comprehensive and accurate identification of eukaryotes and prokaryotes in metagenomic data. bioRxiv. 2019. https://doi.org/10.1101/641332.

13. Clausen P, Aarestrup FM, Lund O. Rapid and precise alignment of raw reads against redundant databases with KMA. BMC Bioinformatics. 2018;19:307.

14. Bidartondo MI. Preserving accuracy in GenBank. Science. 2008;319:1616.

15. Altschul SF, Madden TL, Schaffer AA, Zhang J, Zhang Z, Miller W, Lipman DJ. Gapped BLAST and PSI-BLAST: a new generation of protein database search programs. Nucleic Acids Res. 1997;25:3389-402.

16. Ainsworth D, Sternberg MJE, Raczy C, Butcher SA. K-SLAM: accurate and ultra-fast taxonomic classification and gene identification for large metagenomic data sets. Nucleic Acids Res. 2017;45:1649-56.

17. Longo MS, O'Neill MJ, O'Neill RJ. Abundant human DNA contamination identified in non-primate genome databases. PLoS One. 2011;6:e16410.

18. Breitwieser FP, Pertea M, Zimin AV, Salzberg SL. Human contamination in bacterial genomes has created thousands of spurious proteins. Genome Res. 2019;29:954-60

19. Merchant S, Wood DE, Salzberg SL. Unexpected cross-species contamination in genome sequencing projects. PeerJ. 2014;2:e675.

20. de Goffau MC, Lager S, Salter SJ, Wagner J, Kronbichler A, Charnock-Jones DS, Peacock SJ, Smith GCS, Parkhill J. Recognizing the reagent microbiome. Nat Microbiol. 2018;3:851-3.

\section{Publisher's Note}

Springer Nature remains neutral with regard to jurisdictional claims in published maps and institutional affiliations.

Ready to submit your research? Choose BMC and benefit from:

- fast, convenient online submission

- thorough peer review by experienced researchers in your field

- rapid publication on acceptance

- support for research data, including large and complex data types

- gold Open Access which fosters wider collaboration and increased citations

- maximum visibility for your research: over $100 \mathrm{M}$ website views per year

At $\mathrm{BMC}$, research is always in progress.

Learn more biomedcentral.com/submissions 